\section{Genetic Control of $\beta$-Hydroxybutyric Dehydrogenase in Paramecium aurelia}

Mammalian $\beta$-hydroxybutyric dehydrogenase is known to be firmly bound to the outer membrane of mitochondria and can be extracted by the neutral detergent 'Triton $X \cdot 100^{\prime 1}$. In the investigation reported here the same enzyme was shown-by a similar extraction procedureto be present in the mitochondria of the protozoan Paramecium aurelia. Extracts were made from a number of clones isolated from a natural population of $P$. aurelia (syngen 9), collected near Edinburgh and examined by starch gel electrophoresis. Two electrophoretically distinct forms of the enzyme were found in different clones and have been designated $H B D-1$ and $H B D-2$ respectively (Fig. 1a).

Paramecium aurelia is particularly convenient for the study of the genetics of mitochondrial proteins, for when paramecia undergo conjugation, exchange of haploid nuclei occurs and the two ex-conjugants-each containing cytoplasm (including mitochondria) from one parent only

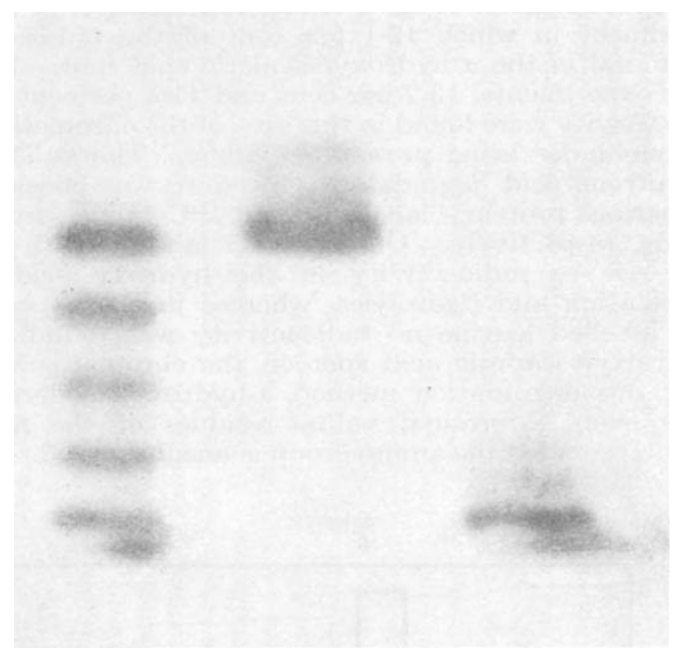

$a$

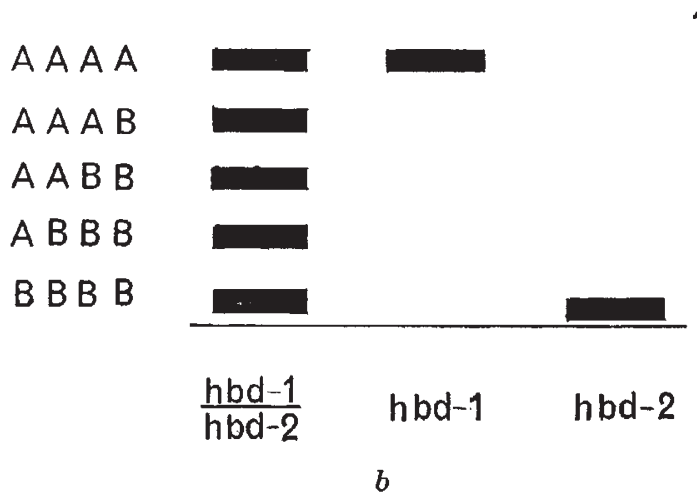

Figs. $1 a$ and $b$. Starch gel zymogram of $\beta$-hydroxybutyric dehydrogenase. Stain developed with $0.5 \mathrm{~g}$ of sodium- $\beta$-hydroxybutyrate, $3 \mathrm{mg}$ of nicotinamide adenine dinucleotide, $6 \mathrm{mg}$ of MTT tetrazolium and $0.5 \mathrm{mg}$
- can be isolated and grown into clones. In this way clones of paramecia are obtained which originate cytoplasmically from one or other of the parent clones, but which contain nuclear genes from both.

The mitochondrial fraction was homogenized in 1 per cent 'Triton $X \cdot 100,{ }^{\prime} 0 \cdot 1 \mathrm{M}$ phosphate buffer $p H \mathrm{H} \cdot 4$, and kept at $4^{\circ} \mathrm{C}$ for $30 \mathrm{~min}$. The supernatant from this extract was then run on a 10 per cent starch gel in a tris-citrate buffer using the discontinuous system of Poulik $^{2}$. Electrophoresis was carried out at $18 \mathrm{v} / \mathrm{cm}$ with the gel at $4^{\circ} \mathrm{C}$ (cooled by an ice-pack), until the brown borate line was 3-4 cm from the anodal wick. The gel was sliced and stained for $\beta$-hydroxybutyric dehydrogenase activity using a method adapted from a histological stain ${ }^{3}$.

Heterozygotes were obtained (from both eytoplasmic origins) by crossing paramecia containing $H B D-1$ and $H B D .2$ respectively. The hybrids containing cytoplasm from either conjugant gave identical patterns on starch gel electrophoresis. This immediately suggests that the genetic factors controlling $\beta$-hydroxybutyric dehydrogenase are nuclear and not cytoplasmic. To confirm this, heterozygotes of the type $H B D-1 / H B D-2$ were passed through autogamy and a $1: 1$ ratio was obtained, indicating the segregation of a pair of alleles corresponding to the two forms of the enzyme (for details of the genetics of $P$. aurelia see ref. 4). These results are shown in Table 1, and establish that this enzyme, although firmly bound to the mitochondrial membrane, is controlled by a nuclear gene.

\section{Table 1 \\ $H B D-1 \quad H B D-2 \quad$ Total \\ $\left(x^{2}=1 \cdot 72\right.$ not significant at the 5 per cent level $)$}

The segregation of the two alleles $H B D-1$ and $H B D-2$, after passing the heterozygote through autogamy.

This raises further questions as to where the polypeptidos are synthesized and how they pass into the mitochondrial membranes. Using isolated mitochondria, Roodyn, Work and Reis ${ }^{5}$ found incorporation of radioactive amino-acids into a 'Triton $X$-100' insoluble fraction; this would suggest that synthesis of proteins soluble in 'Triton $X-100$ ' occurs outside the mitochondrion. This means that the polypeptides must be assembled and incorporated into the membrane from outside.

A further piece of information which has come from this work is that $\beta$-hydroxybutyric dehydrogenase is a tetramer, consisting of identical polypeptide chains. In the homozygous state the enzyme occurs as a single band, for all the polypeptides are identical, whereas in the heterozygous state a five banded pattern is obtained. This is easily explained if $H B D .1$ consists of four polypeptides AAAA and $H B D-2$ consists of BBBB (A and $\mathrm{B}$ differing in charge); the heterozygote will then contain a randomly assorted mixture of the two types ${ }^{6}$ as shown in Fig. $1 b$.

I thank Professor G. H. Beale for help and encouragement. This work was carried out during the tenure of a Medical Research Council postgraduate scholarship.

\section{A. TAIT}

Protozoan Genetics Unit,

Institute of Animal Genetics,

University of Edinburgh.

Received June 17, 1968.

Allmann, D. W., Bachmann, E., and Green, D. E., Arch. Biochem. Biophys., $115,165(1966)$

2 Poulik, M. D., Methods in Biochemical Analysis, 14, 455 (1966).

${ }^{3}$ Seligmann, A. M., Methods in Enzymology, 6, 892 (1963).

4 Beale, G. H., The Genetics of Paramecium aurelia (Cambridge University Press, 1954)

${ }^{5}$ Roodyn, D. B., Work, T. S., and Reis, P. J., Biochem. J., 80, 9 (1961).

- Shaw, C. R., Brookhaven Symposia in Biology, 17, 117 (1964). 